\title{
The Formation of Chemical Synapses Between Cell-Cultured Neuronal Somata
}

\author{
P. G. Haydon \\ Department of Zoology, lowa State University, Ames, lowa 50011
}

The study of the development and plasticity of chemical synaptic connections is frequently restricted by the lack of access to the synaptic terminals. This can, in part, be overcome by plating neurons into cell culture where all regions of a neuron are made experimentally accessible. However, the small size of synaptic terminals still makes direct experimental manipulation difficult. In this study we have found, in the absence of neurite extension, directly contacting cell somata (diameter $50-100 \mu \mathrm{m}$ ) will form chemical synapses. Identified neurons B5 and B19 of Helisoma were plated into culture under conditions that promote adhesion between cell pairs. Under these conditions, neurite outgrowth was absent, but action potentials in B5 evoked inhibitory postsynaptic potentials in B19 that were reversed in sign by the injection of chloride ions and were blocked by tubocurare $\left(10^{-5} \mathrm{M}\right)$, reduced extracellular $\mathrm{Ca}^{2+}$, and $\mathrm{Cd}^{2+}$ ions. Such synapses exhibited classical properties of chemical synapses, including the spontaneous release of neurotransmitter.

Since somatic synapses represent an appropriate model of synaptic transmission, this system was utilized to study the role of mutual neuronal contact in the development of transmitter release capabilities. Future pre- and postsynaptic somata were cultured separately for $3 \mathrm{~d}$, the period required for the development of synaptic transmission under conditions of maintained contact. Then, neurons were made to contact and intracellular recordings taken within 0 to 4 hours. Postsynaptic potentials were detected as early as 10 sec following contact. Thus, qualitatively the development of transmitter release capabilities does not require maintained contact. The ability to form synapses between directly contacting spherical somata now permits the study of both mature excitation-secretion coupling and the development of synaptic transmission at a new level of resolution since both the pre- and postsynaptic elements of a neuronal synapse are directly accessible to experimental manipulation and control.

Recent work on the development of synaptic connections has increased our understanding of the events leading to the formation of functional synaptic transmission. Motile growth cones contain and can release neurotransmitter (Hume et al., 1983; Young and Poo, 1983). Contact with muscle induces further

\footnotetext{
Received May 4, 1987; revised Aug. 24, 1987; accepted Sept. 10, 1987.

This work was supported by PHS Grant NS24233. I wish to thank Drs. C. Drewes, R. G. Wong, and M. Zoran for critical comments on the manuscript.

Correspondence should be addressed to P. G. Haydon at the above address.

Copyright $(C) 1988$ Society for Neuroscience $0270-6474 / 88 / 031032-07 \$ 02.00 / 0$
}

release of neurotransmitter from the growth cone, and within seconds of contact, miniature postsynaptic currents can be detected in the postsynaptic muscle cell (Xie and Poo, 1986). However, such transmission does not represent the fully differentiated synaptic connection. For example, after the presynaptic neurite contacts the muscle membrane, alterations in the spatial distribution of postsynaptic AChRs occur (for review, see Schuetze and Role, 1987). Although such studies have provided insight into the development of synaptic transmission, little is known about the physiological development of the presynaptic terminal. The small size of synaptic terminals has made it difficult to directly record and control presynaptic membrane potential during development, as has proved possible in a limited number of mature preparations (Auerbach and Bennett, 1969; Martin and Ringham, 1975; Llinás et al., 1981a, b; Wojtowicz and Atwood, 1984; Lemos et al., 1986). With the advent of patch-pipettes, however, it has been possible to study growth cones in cell culture. For example, using cell-attached and wholecell recording techniques, it has been determined that growth cones contain a variety of voltage-gated ion conductances (Cohan et al., 1985; Belardetti et al., 1986a, b) which share certain properties with the membrane of neuronal somata (Belardetti et al., 1986b). However, it has not yet been possible to determine the developmental events within the presynaptic tcrminal following contact with the postsynaptic cell.

Identified neurons of leech (Fuchs et al., 1981, 1982; Henderson, 1983; Henderson et al., 1983; Arechiga et al., 1986), Aplysia (Camardo et al., 1983; Bodmer et al., 1984; Schacher et al., 1985), and Helisoma (Hadley et al., 1983, 1985) have been used in cell-culture studies of electrical and chemical synaptogenesis. Isolated neurons plated in cell culture extend neurites and form synaptic connections. An attractive feature of the leech cell-culture system is that neurons extend short neurites and, as a consequence, microelectrode recordings made from the cell body are electrically close to the synaptic terminals (Fuchs et al., 1982; Pellegrino and Simonneau, 1984; Dietzel et al., 1986). While neuronal processes characteristically form the presynaptic elements of synaptic profiles, recent evidence suggests that nerve cell bodies may be accessible models for studying presynaptic function. For example, denervated avian ciliary ganglia release $\mathrm{ACh}$ in response to $\mathrm{K}^{+}$depolarization, antidromic stimulation, and cholinergic agonists; this led the authors to conclude that neurotransmitter is released from somata (Johnson and Pilar, 1980). Additionally, Chow and Poo (1985) demonstrated that spontaneous miniature potentials were detected in myoblasts following contact with neuronal somata. Taken together, these observations raise the possibility that a pair of neuronal cell bodies could form a chemical synapse. 


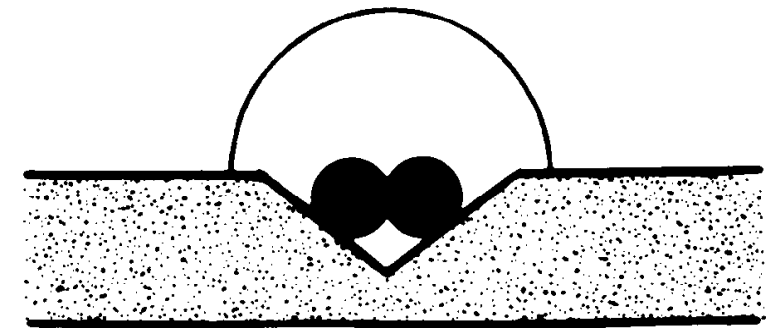

Figure 1. Schematic illustrating the technique used to cause contact and, subsequently, adhesion between pairs of neurons.

In this report a test is made of the hypothesis that in the absence of neurite extension neuronal cell bodies are competent to form chemical synaptic connections. Specifically, identified neurons B5 and B19 of Helisoma were utilized; these neurons have previously been shown to form a unidirectional chemical synapse in cell culture when contact is made through newly extended neurites (P. G. Haydon and S. B. Kater, unpublished observations). This study demonstrates that neuronal somata readily form chemical synaptic connections, and thcir propcrtics parallel those of the more classical synapses that are located on neuronal processes. Additionally, by manipulating the timing of initial contact between future pre- and postsynaptic partner somata, the role of contact in regulating the development of transmitter release properties is examined.

\section{Materlals and Methods}

In all experiments, adult specimens of the albino strain (red) of the pond snail Helisoma were used. Animals were laboratory-reared, maintained in aquaria, and fed lettuce and trout chow. As described in detail previously (e.g., Wong et al., 1981; Haydon et al., 1985), snails were deshelled, and the paired buccal ganglia were acutely isolated from individual snails in antibiotic saline (ABS). Paired buccal ganglia were treated with a $0.2 \%$ solution of trypsin (Sigma, type III) in defined medium (DM; 50\% Leibowitz-15 (GIBCO) with Helisoma salts) for $20-45 \mathrm{~min}$ followed by $15 \mathrm{~min}$ in $0.2 \%$ trypsin inhibitor (Sigma type II-S). Using sterile procedures, enzymatically treated ganglia were pinned to the silicon rubber floor of a dissection dish for single-cell excision. Using an electrolytically sharpened tungsten microknife, a small slit was made in the connective sheath adjacent to the visually identified neuronal cell body. By applying gentle pressure to the ganglion, the cell of choice "popped" out through the slit, and by applying negative pressure through a glass suction pipette, the neuronal soma and attached proximal axon were reliably removed from the ganglionic environment (Haydon et al., 1985).

Cell culture. Neurite extension critically relies on adhesion to an appropriate substratum. Thus, to prevent neurite extension, neurons were plated into a nonadhesive culture environment. Nonfiltered snail hemolymph when applied to a culture dish reliably prevents cell adhesion to the substrate. Hemolymph was extracted from snails on the day of culture, as described by Hadley and Kater (1983). Neurons were plated into a $40 \mu \mathrm{l}$ droplet of a $50 \%$ solution of hemolymph in DM. To optimize contact and subsequent adhesion between cell pairs, a depression was made in the base of a Falcon \#1008 petri dish into which neuron pairs were plated (Fig. 1). In this manner, greater than $95 \%$ of cell pairs adhered to one another and never to the substrate. After a period of 4$9 \mathrm{hr}$, cell pairs were removed from this droplet, using a suction pipette, and transferred to $2 \mathrm{ml}$ of a $1 \%$ solution of hemolymph in DM. For prolonged culture, hemolymph was changed every 3-4 d.

Electrophysiology. In order to test for synaptic connectivity, neuronal cell pairs were transferred to a Falcon $\# 1008$ petri dish containing DM or, more characteristically, to a polylysine-coated Falcon \#3001 culture dish (Wong et al., 1981), where they rapidly adhere to the substrate. Such immobilized cells were penetrated with glass microelectrodes filled with either $2 \mathrm{M}$ potassium acetate (KAc) or with $1.5 \mathrm{M}$ potassium chloride (see Results) and had DC resistances in the range of 8-25 M 2 . Standard electrophysiological techniques were utilized throughout. Tu- bocurarine chloride (Sigma) was dissolved in standard Helisoma saline for application to cultured cells. ACh (Sigma) was focally applied to neuron B19 using positive-pressure ejection from a micropipette.

Neuronal morphology. The morphology of neuron pairs was determined by phase-contrast optics using a Nikon Diaphot inverted microscope. To further test for the presence of neurites extending from a neuronal cell body the fluorescent dye Lucifer yellow was iontophoretically injected into one neuron of a cell pair as described previously (Cohan et al., 1987). Neuronal morphology was subsequently ascertained using fluorescence optics of the Nikon Diaphot on living tissue. In control experiments where neurons were plated into culture under conditions that promote neurite extension, intrasomatically injected Lucifer yellow reliably filled neurites over distances in excess of $1 \mathrm{~mm}$, and, when neurites passed over adjacent cell bodies, these processes were readily discernible with this dye.

\section{Results}

\section{Morphology}

Neurons within the nervous system of Helisoma are identifiable on the bases of visual criteria, which include cell size, coloration, and position within the ganglion. Isolated neuron pairs plated onto an adhesive substrate for recording retained their identifiability on the bases of 2 of these criteria: (1) neuron B5 is larger than neuron B 19 (Fig. 2), and (2) B5 contains a prominent region of pigmentation. Subsequent penetration with intracellular microelectrodes confirmed cell identity, since the duration of neuron B19's action potential is shorter than that of B5 (Cohan et al., 1985).

Using phase-contrast optics, cell pairs appeared as spherical structures devoid of neurites. However, to confirm that cell interactions were mediated directly by somata, the fluorescent dye Lucifer yellow was iontophoretically injected into the soma of one neuron of a pair. Subsequent visualization using fluorescent optics never revealed the presence of neurites overlying the membrane of the adjacent paired cell. Each injected neuron had the appearance of a sphere (Fig. 2). From dye injections made on days $2-7$ of culture, neurites were never detected $(n=$ 7), indicating that neurons B5 and B19 make direct contact by their cell somata. Furthermore, examination of serial sections of cell pairs with transmission electron microscopy has not revealed the presence of neurites extending from one cell over the other (Emery and Haydon, unpublished observations).

\section{Synaptic connectivity}

Since adhesion between directly apposed somata is maintained in the absence of neurite extension, the state of connectivity of such neuron pairs was determined. At this time, the presence of both electrical and chemical synapses was tested.

Hyperpolarizing current was injected into either B5 or B19, and the response in the paired neuron was determined to assay for electrical coupling. In $63 \%$ of preparations DC current did not pass between cell pairs ( $n=22$ of 35 ; days $1-8$; Fig. 3), although in remaining preparations electrical connections did form (e.g., Fig. 6). By contrast, intracellular recordings revealed the reliable presence of a unidirectional chemical synapse.

The connectivity of paircd ncurons was tested on days 1-8 of culture. On days 1-2 of culture chemical synaptic connections were found in 1 of 7 pairs examined. After a latent period of 3 $\mathrm{d}$, however, action potentials in neuron B5 evoked synaptic responses in $\mathrm{B} 19$ in $100 \%$ of cell pairs tested (Fig. $3 ; n=28$, days 3-8 of culture), with latencies in the range of $0.4-9.3 \mathrm{msec}$ $(n=7$; mean $=4.6 \mathrm{msec})$. Chemical synapses formed irrespective of whether a conjoint electrical connection was present. Initial recordings were made from cell somata using $2 \mathrm{~m}$ potas- 


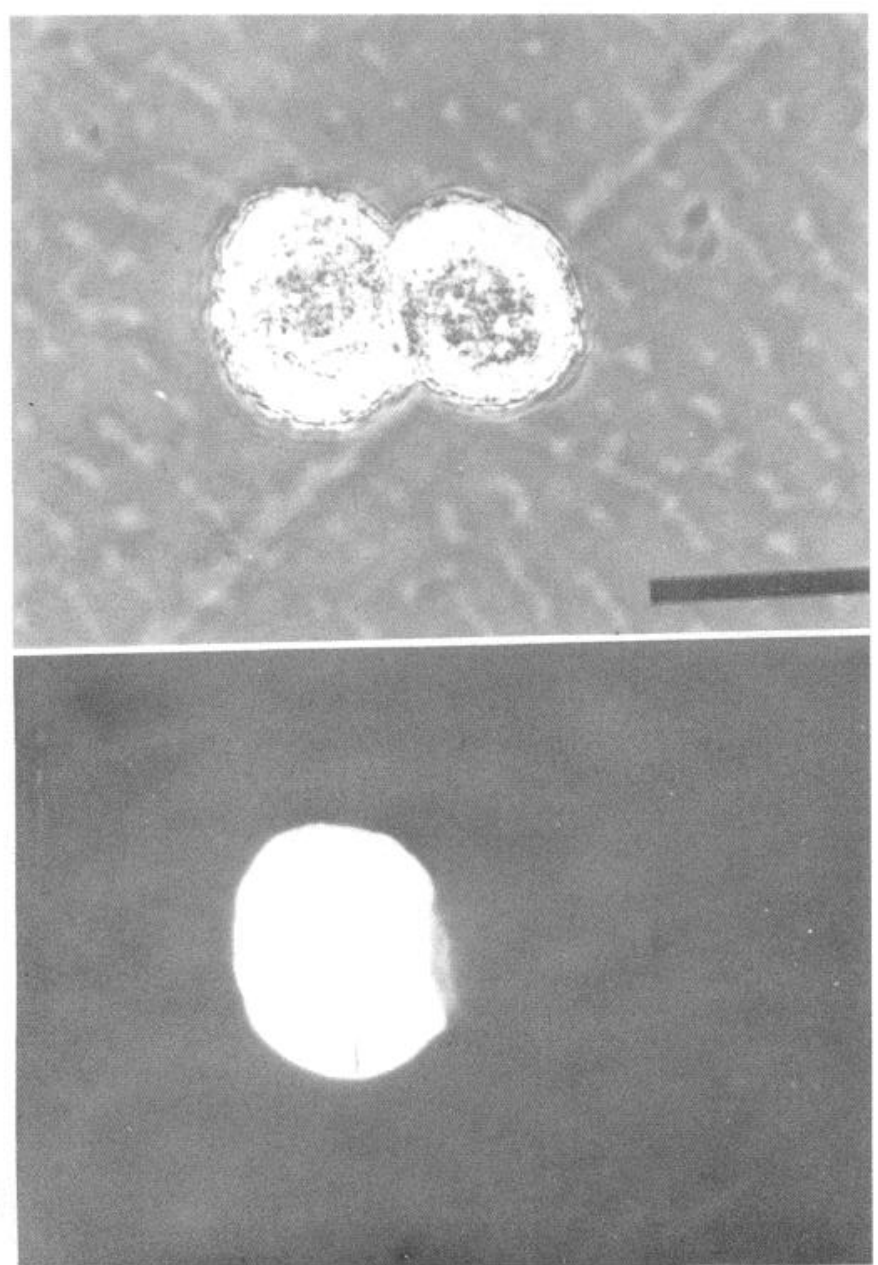

Figure 2. Neuron pairs maintain a spherical structure in cell culture. Cell pairs maintained contact for the total duration of culture and, when viewed with phase-contrast optics (top: B5, left; B19, right), were seen to adopt a characteristic spherical morphology. The injection of Lucifer yellow into neuron B5 confirmed that somata did not extend neurites (bottom). Scale bar, $50 \mu \mathrm{m}$.

sium acetate-filled glass microelectrodes. Under such conditions, action potentials in B5 evoke inhibitory synaptic responses in B19, which reversed in sign by hyperpolarization of the postsynaptic membane potential (Fig. 3). In previous experiments performed on cells with neurites, we observed that impaling B19 with a $\mathrm{KCl}$-filled electrode reversed the polarity of the B5 evoked ipsp recorded in B19 (P. G. Haydon and S. B. Kater, unpublished observations). Therefore, we determined whether $\mathrm{KCl}$ electrodes had similar effects on the somatic ipsp. Penetration of $\mathrm{B} 19$ with a $\mathrm{KCl}$-filled microelectrode caused a rapid reversal of the evoked ipsp (Figs. 4-7). These data indicate that the synapse which forms between contacting somata has the same properties as the connection between newly extended neurites, and that the synaptic potential is mediated, at least in part, by chloride ions. For the remainder of this study, $\mathrm{KCl}$ filled microelectrodes were utilized since, in addition to reversing the sign of the synaptic potential, its amplitude was augmented.

The chemical connection that forms between neurites of B5 and B19 is believed to be cholinergic (P. G. Haydon and S. B. Kater, unpublished observations). To further substantiate the chemical nature of synaptic transmission between pairs of cell

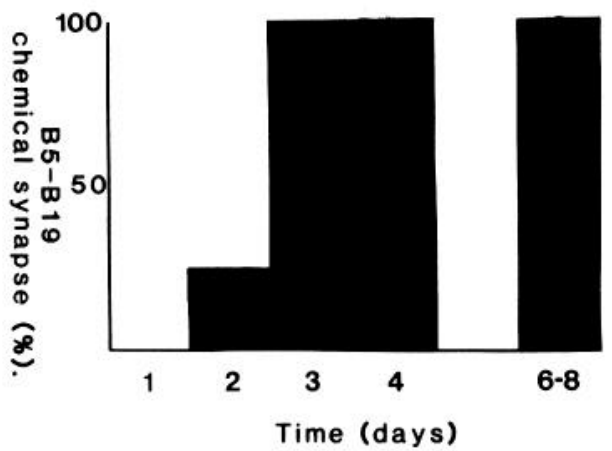

B19

B5

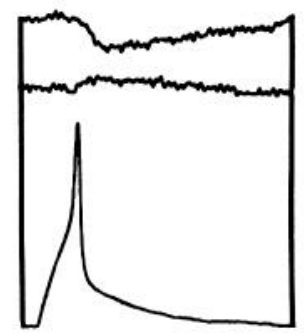

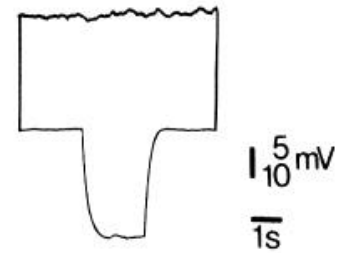

$\overline{200 \mathrm{~ms}}$
Figure 3. Isolated neuronal somata form chemical synaptic connections in cell culture. Intracellular recordings made from neurons B5 and B19 demonstrate that somata form chemical synaptic connections. Bottom, Action potentials in B5 evoke inhibitory synaptic potentials in B19 (left), which are reversed in sign with hyperpolarization of B19's membrane potential (KAc-filled microelectrodes). In this cell pair, DC current did not pass between neuronal somata, indicating that they were not electrically coupled (right). Top. Time course of formation of the B5-B19 somatic chemical synapse. Neuron pairs were removed from culture on days $1-8$ of culture and the state of connectivity was examined $(n=35)$. This chemical synapse is first reliably detected on day 3 of culture and is maintained throughout the experimental period of $8 \mathrm{~d}$.

somata and to test the cholinergic nature of such transmission, we performed the following tests: tubocurarine chloride was added to the bathing medium of synaptically connected cell pairs and $\mathrm{ACh}$ was focally applied to the membrane of B19. Tubocurare $\left(10^{-5} \mathrm{M}\right)$ reliably reduced the amplitude of the B5 evoked synaptic action in $\mathrm{B} 19(n=4$; Fig. 4). Such data implicate ACh as being the neurotransmitter utilized at this synapse. This is further corroborated by the application of $\mathrm{ACh}$ to the membrane of B19 (days 0-3). When using a potassium acetate electrode to record from B19, ACh applied to B19 evoked a hyperpolarization that was reversed in direction when B19 was hyperpolarized by DC current injection. When using a $\mathrm{KCl}$-filled electrode to record from $\mathrm{B} 19$, ACh evoked a large depolarization of B19 ( $n=4$; Fig. 4). Furthermore, tubocurarine $\left(10^{-5} \mathbf{M}\right)$ reversibly reduced the amplitude of such $\mathrm{ACh}$-evoked depolarizations $(n=3)$. Taken together, such evidence indicates that neuron B5 forms a cholinergic chemical synapse with B19 when neuronal somata are in direct contact.

Intracellular recordings made from B5 and B19 have demonstrated that neurotransmitter can be released spontaneously. At resting potential, the isolated soma of B5 did not discharge spontaneous action potentials. However, simultaneous recordings made from B19 demonstrated the presence of spontaneous postsynaptic potentials (Fig. 5). Depolarization of B5 increased the frequency of such psps. The injection of depolarizing current 
Tubocurare
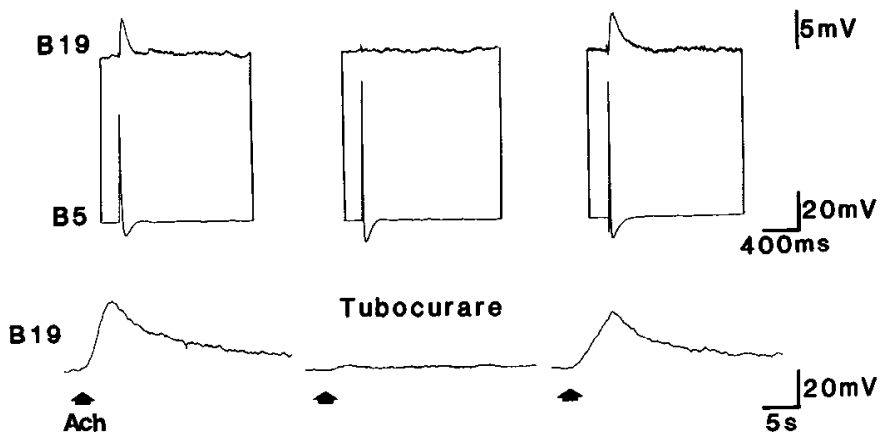

Figure 4. The synaptic connection that forms between somata of B5 and B19 is sensitive to tubocurare. Single action potentials in B5 evoke excitatory synaptic potentials in B19 when the postsynaptic neuron was impaled with a $\mathrm{KCl}$ electrode (upper left). The addition of $10^{-5} \mathrm{M}$ tubocurare to the bathing saline reduced the amplitude of the B5 evoked synaptic potential (middle), which was reversed upon washout of this cholinergic antagonist (right). Pipette application of ACh to B19 (lower left) evoked a similar depolarization of $\mathrm{Bl} 9$ (KCl electrode), which was also reversibly reduced in amplitude by application of $10^{-5} \mathrm{M}$ tubocurare.

into B5 evoked a single action potential and associated evoked synaptic potential in B19. Frequently, maintained depolarization of B5 failed to evoke additional action potentials. Under these conditions of sustained depolarization, however, a barrage of desynchronized psps was detected in B19 (Fig. 5).

\section{$\mathrm{Ca}^{2+}$-dependent transmitter release}

Neurotransmitter release at synaptic terminals is dependent on an influx of $\mathrm{Ca}^{2+}$. The calcium dependence of transmitter release in this system of neuronal somata was demonstrated by the use of a low-Ca ${ }^{2+}$ saline and saline containing the $\mathrm{Ca}$ channel blocker $\mathrm{Cd}^{2+}$ (Kostyuk, 1980). The concentration of extracellular $\mathrm{Ca}^{2+}$ was reduced from 4.1 to $0 \mathrm{mM}$, while the $\mathrm{Mg}^{2+}$ concentration was increased by $4.1-5.6 \mathrm{~mm}$, or $\mathrm{CCl}_{2}$ was added to normal saline (final concentration, $1 \mathrm{~mm}$ ) to block $\mathrm{Ca}^{2+}$ currents. Figure 6 demonstrates that nominally calcium-free saline blocks activity-dependent transmission, and the addition of $\mathrm{Cd}^{2+}$ to the bathing saline blocks desynchronized transmission in response to presynaptic DC depolarization.

Taken together, the experiments described above demonstrate that, in the absence of neurites, neuronal somata are capable of forming functional chemical synaptic connections. Therefore, in order to address questions concerning the formation of synaptic connections, neuronal somata act as an experimentally accessible model for developing synaptic terminals.

Do the fundamental features of the synapse require maintained contact between B5 and B19 for their development? To address this question, neurons B5 and B19 were isolated from the nervous system and immediately placed into separate culture dishes ( $2 \mathrm{ml} \mathrm{1 \%}$ hemolymph). Then, after a period of $3 \mathrm{~d}$, which corresponds to the latent period for synaptogenesis (Fig. 3), either B5 or B19 was transferred to the partner neuron's culture dish so that contact was made between cell pairs. Immediately upon contact, cells strongly and irreversibly adhered to one another, permitting the subsequent transfer of the cell pair into a standard polylysine-coated recording chamber. Following transfer, cells
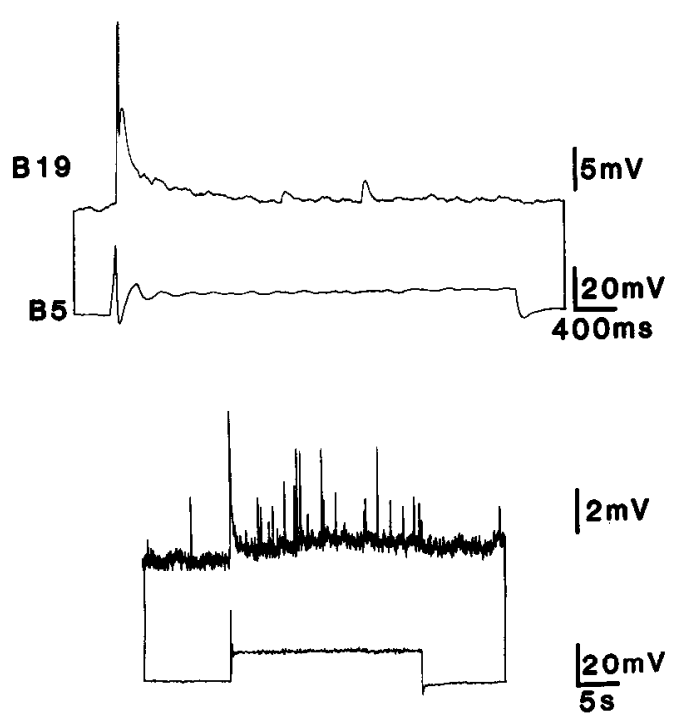

Figure 5. Synaptic potentials in B19 detected in the absence of action potentials in B5. Prolonged depolarization of B5 evoked a single action potential, which was followed by a maintained depolarization of the cell. The action potential in B5 evoked a single, large-amplitude synaptic potential in B19, which was followed by smaller amplitude synaptic potentials (upper traces). Furthermore, spontaneous synaptic potentials could be detected in B19 in the absence of action potentials and depolarization of B5 (lower traces). The maintained depolarization of B5 increased the frequency of such synaptic potentials that are composed of multiple amplitude events (note different time scale between upper and lower traces).

became immobilized and were impaled with microelectrodes within minutes, or up to $4 \mathrm{hr}$ following contact, in order to determine connectivity.

Intracellular recordings made from neurons that first contact on day 3 demonstrate that maintained contact for the total culture period is not required for the development of functional synaptic transmission. Chemical psps were detected in $\mathrm{B} 19(n=$ 8 of 10 cells examined; Fig. 7) as early as 12 min after contact (earliest time of recording). To make a more appropriate determination of the time of contact required until detection of functional transmission, additional neurons B5 and B19 were separately impaled with a microelectrode and micromanipulated to make contact $(n=3)$ while intracellular records were being taken. In this manner, the earliest evoked psp was detected $10 \mathrm{sec}$ following contact. By contrast, electrical connections did not form either when cells contacted while intracellular records were being taken ( $n=3$ ) or when they had contacted for up to $4 \mathrm{hr}$ before recordings were taken ( $n=10$; Fig. 7).

The functional chemical connection had similar properties to the same connection that forms under conditions of maintained contact: spontaneous, evoked (Fig. 7) and desynchronized release of neurotransmitter were detected shortly after contact. Additionally, the evoked psps followed presynaptic action potentials with a short latency, as is characteristic of cell pairs cultured under conditions of prolonged contact. This is not to say, however, that prolonged contact has no effect on the development of the presynaptic transmitter release capabilities. Neurons that contact immediately before recordings are made were capable only of the intermittent release of transmitter. That is, psps detected in B19 did not follow presynaptic action potentials one for one. 
a

OCa

Figure 6. Transmitter release from the soma of neuron $\mathrm{B} 5$ is $\mathrm{Ca}^{2+}$ dependent. a, Burst of action potentials in B5 evoked multiple psps in B19 when bathed in standard snail saline (left). However, exchanging the saline to a 0 $\mathrm{Ca}^{3+}$, high- $\mathrm{Mg}^{3+}$ saline rapidly abolished synaptic transmission (right). $b$, DC depolarization of $\mathrm{B} 5$ evoked a maintained release of transmitter in the absence of presynaptic action potentials when bathed in standard snail saline $($ left $)$. Note that 4 of these psps evoked action potentials in B19 that are clipped by the pen-recorder. Addition of $1 \mathrm{~mm}$ $\mathrm{Cd}^{2+}$ to this standard saline rapidly abolished all transmitter release from the sama of B5. The residual DC coupling between $\mathrm{B} 5$ and $\mathrm{B} 19$ was due to the presence of an electrical connection.

\section{Discussion}

There is a striking similarity between the properties of the somatic synapse that forms in cell culture and connections that are normally present in vivo at synaptic terminals. For example, synaptic transmission is $\mathrm{Ca}^{2+}$-dependent. Transmitter can be released spontaneously. The DC depolarization of the presynaptic soma increases desynchronized transmitter release. Action potentials evoke transmitter release. The action of the neurotransmitter is blocked by a postsynaptic antagonist. Given our knowledge of synaptic function, these properties are to be expected. However, the ability to control the formation of such chemical synapses at this isolated, experimentally accessible site in a predictable way readily permits the study of the fundamental mechanisms of synaptic transmission that have, to date, not been possible in more intact systems.

In addition to questions concerning the mechanisms of function of a mature synapse are questions concerning the control of synaptic development. Toward this goal, much experimental attention has focused on elucidating the control of the spatial arrangement of postsynaptic AChRs (for review, see Schuetze and Role, 1987). The transformation of the growth cone into the presynaptic terminal has been less intensively studicd. However, it is known that growth cones of developing neurons can release neurotransmitter prior to contact by postsynaptic targets (Hume et al., 1983; Young and Poo, 1983), and subsequently, contact with the muscle target induces further release of neurotransmitter within the early minutes of contact (Xie and Poo, 1986). Using somata it has now been possible to determine whether similar properties exist for regenerating neurons. These results demonstrate that when maintained for $3 \mathrm{~d}$ following
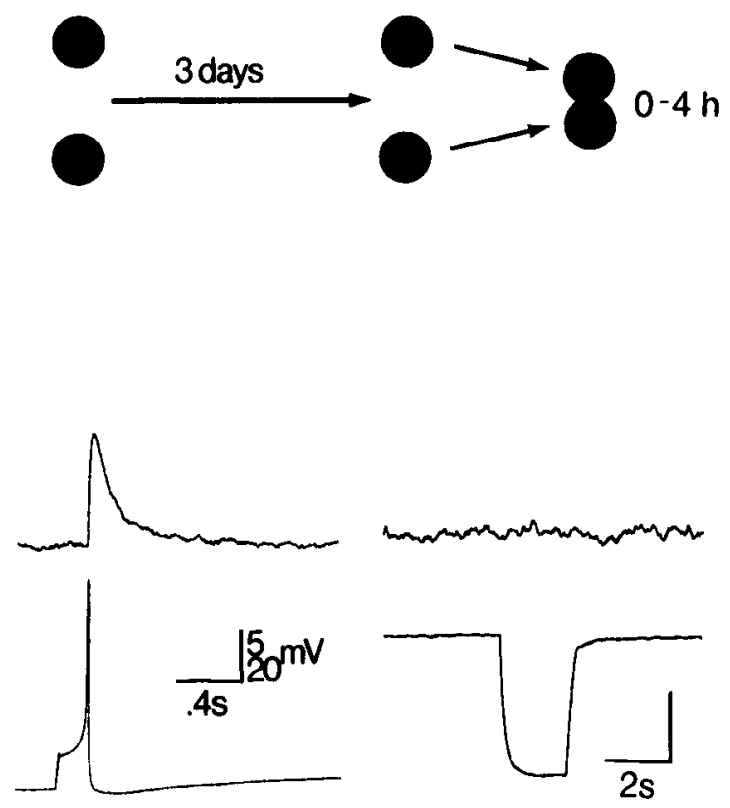

Figure 7. Maintained contact between pre- and postsynaptic neurons is not required for the development of transmitter release capabilities. Neurons B5 and B19 were plated separately into culture. After a period of $3 \mathrm{~d}$, contact was induced between cell pairs and the ability to release neurotransmitter from the adult soma of B5 tested. Left, Neurotransmitter release was reliably detected using B19 as a bioassay, and action potentials in B5 could evoke postsynaptic responses. Right, By contrast, the injection of DC current into either B5 of B19 failed to evoke a synchronous potential change in the partner neuron, indicating that detectable electrical connections do not form within $4 \mathrm{hr}$ of contact. (Different neuron pairs used in each set of traces.) 
axotomy, the normal period for synaptogenesis in cell culture, adult neurons have similar capabilities to developing neurons. Within the first minutes of contact between pre- and postsynaptic neurons, transmitter release is readily detected. Thus, just as in development, maintained contact between future pre- and postsynaptic targets is not required for the development of the initial transmitter release capability.

A surprising observation in our experiments was the variability of synaptic delay. Since action potentials were evoked at the site of transmitter release, we would expect a short synaptic delay. In the squid giant synapse, transmitter release occurs after a delay of approximately $200 \mu \mathrm{sec}$ following the influx of calcium. In our experiments, however, latencies ranged from 400 $\mu \mathrm{sec}$ to $9.3 \mathrm{msec}$. While several interpretations are possible, the variability and long delay of some preparations may result from the fact that these cells were at different stages in development.

In vivo neurons $\mathrm{B} 5$ and $\mathrm{B} 19$ are not synaptically interconnected, but, in response to axotomy, this neuron pair is able to form novel chemical and electrical connections. As demonstrated previously, neurons B5 and B19 will form an electrical connection in situ (Hadley and Kater, 1983) and in cell culture (Haydon et al., 1984, 1987). These previous studies did not report the formation of a novel chemical connection. However, these in situ experiments utilized high- $\mathrm{Mg}^{2+}$ saline to block the release of neurotransmitter, so that a higher-resolution analysis of electrical connections could be performed. When using $\mathrm{Ca}^{2+}$ containing saline we have now determined that a novel chemical connection can form between B5 and B19 in situ (P. Matthews and P. G. Haydon, unpublished observations). Additionally, these same neurons can form a novel chemical connection in cell culture under conditions where contact is made through newly extended neurites (P. G. Haydon and S. B. Kater, unpublished communications). The chemical connection that forms between $\mathrm{B} 5$ and $\mathrm{B} 19$, when contact is made through neurites, has the same properties as the somatically mediated connection reported here: The connection is unidirectional, B5 being presynaptic to B19; psps are blocked by tubocurare; and B5 evokes ipsps in B19 that are reversed in sign by penetration of B19 with a $\mathrm{KCl}$-filled electrode. Thus, while certain neurons re-form only appropriate chemical synapses in cell culture (Fuchs et al., 1981, 1982; Camardo et al., 1983), the formation of a novel B5-B19 chemical synapse is an additional example of a neuron that forms connections not normally found in the nervous system (Bodmer et al., 1984; Schacher et al., 1985).

The focus of much of the previous work on neurons B5 and B19 of Helisoma has been on the conditions required for the formation of electrical connections. Those studies demonstrated that electrical connections form under conditions of synchronous neurite extension (Hadley et al., 1983, 1985). If one cell of a pair has ceased extension, electrical connections do not form. Since neuronal somata do not extend neurites, it might be surprising that electrical connections did form in $37 \%$ of our cell pairs. However, since previous studies examined when an isolated neuron loses its competence to form an electrical connection, it is not known when a neuron gains the ability to form this type of connection-before neurite extension commences or thereafter. Our data suggest that somata do have a limited capacity to form electrical connections; however, electrical connections form reliably only under conditions of active neurite extension.

Synaptic terminals in vivo are located in specific spatial locations of a given neuron. A fundamental question concerns the nature of the signals and the mechanisms involved in controlling the appropriate spatial location of synaptic terminals. A priori it might be reasonable to assume that the specific location of a synapse is not determined solely by presynaptic intrinsic signals, but rather by a balance of signals arising from pre- and postsynaptic cells upon contact. In this way, the site of contact determines the synaptic site, rather than the postsynaptic cell having to seek a predetermined position of presynaptic membrane. This is certainly the strategy utilized for the development of the postsynaptic membrane of the neuromuscular junction, where the presynaptic growth cone induces the localized aggregation of postsynaptic AChRs at the site of contact. Consonant with this theme, Chow and Poo (1985) demonstrated that the ability to release neurotransmitter is not spatially restricted. Using a myoblast to detect neurotransmitter, they found that growth cone, neurite, and soma can release neurotransmitter. Furthermore, the demonstration that neuronal somata of Helisoma reliably form chemical synapses lends support to the notion that all neuronal regions are competent to form synaptic connections. However, if all regions are competent, why do somata rarely become presynaptic elements of a synapse? Several mechanisms could exert spatial regulation over synaptogenesis. Signals that control the direction of neurite extension and glial elements that encapsulate neuronal somata of Helisoma (Berdan and Bulloch, 1986) would reduce the incidence of neurite-soma contact. Alternatively, while all regions are able to form a synapse, separate regions of a single neuron may compete for the ability to form a functional release site. Such a scheme might be suggested by the demonstration that transmitter release from somata is prevented if the neurites of the same ncuron have previously contacted a myoblast (Chow and Poo, 1985).

When adult Helisoma neurons are excised and plated together as pairs, synaptic connections are first detected after a latent period of $3 \mathrm{~d}$. Because of the accessibility of this synapse, it should be possible to determine the developmental sequence of events underlying this latent period. Application of $\mathrm{ACh}$ to neuron B19 (days 0-3) demonstrated that postsynaptic receptors are present throughout the early stages of contact prior to the detection of functional synaptic transmission. Such lines of evidence point toward a presynaptic locus for the rate-limiting step(s) in the development of functional transmission. Mature synaptic transmission consists of a cascade of events triggered by membrane depolarization. Since the synaptic terminal was experimentally depolarized in the first days following contact and transmitter release was not detected, these data point toward at least 1 of 3 loci for the rate-limiting step in synaptic development: the appearance of a $\mathrm{Ca}^{2+}$ conductance, the synthesis of neurotransmitter, or the development of coupling between a calcium influx and transmitter release. Since we are able to voltage-clamp the presynaptic soma directly and to inject molecules presynaptically, this system should permit the elucidation of the development of each of these critical components to functional transmission.

\section{References}

Arechiga, H., M. Chiquet, D. P. Kuffler, and J. G. Nicholls (1986) Formation of specific connections in culture by identified leech neurones containing serotonin, acetylcholine and peptide transmitters. J. Exp. Biol. 126: 15-31.

Auerbach, A. A., and M. V. L. Bennett (1969) Chemically mediated transmission at a giant fiber synapse in the central nervous system of a vertebrate. J. Gen. Physiol. 53: 183-210. 
Belardetti, F., S. Schacher, E. R. Kandel, and S. Siegelbaum (1986a) The growth cones of Aplysia neurons: Modulation by serotonin of action potential duration and single potassium channel currents. Proc. Natl. Acad. Sci. USA 83: 7094-7098.

Belardetti, F., S. Schacher, and S. Siegelbaum (1986b) Action poten tials, macroscopic and single channel currents recorded from growth cones of Aplysia neurones in culture. J. Physiol. (Lond.) 374: 289313.

Berdan, R. C., and A. G. M. Bulloch (1986) Gap junction specificity in Helisoma ganglia. Neurosci. Abstr. 12: 539.

Bodmer, K., D. Dagan, and I. B. Levitan (1984) Chemical and electrotonic connections between Aplysia neurons in primary culture. $\mathbf{J}$. Neurosci. 4: 228-233.

Camardo, J., E. Proshansky, and S. Schacher (1983) Identified Aplysia neurons form specific chemical synapses in culture. J. Neurosci. 3: 2614-2620.

Chow, I., and M.-M. Poo (1985) Release of acetylcholine from embryonic neurons upon contact with muscle cell. J. Neurosci. 5: 10761082.

Cohan, C. S., P. G. Haydon, and S. B. Kater (1985) Single channel activity differs in growing and non-growing growth cones of isolated identified neurons of Helisoma. J. Neurosci. Res. 13: 285-300.

Cohan, C. S., P. G. Haydon, A. J. Mercier, and S. B. Kater (1987) Formation, maintenance, and functional uncoupling of connections between identified Helisoma neurons in situ. J. Neurobiol. 18: 329341.

Dietzel, I. D., P. Drapeau, and J. G. Nicholls (1986) Voltage dependence of 5-hydroxytryptamine release at a synapse between identified leech neurones in culture. J. Physiol. (Lond.) 372: 191-205.

Fuchs, P. A., J. G. Nicholls, and D. F. Ready (1981) Membrane properties and selective connexions of identified leech neurones in culture. J. Physiol. (Lond.) 316: 203-223.

Fuchs, P. A., L. P. Henderson, and J. G. Nicholls (1982) Chemical transmission between individual retzius and sensory neurones of the leech in culture. J. Physiol. (Lond.) 323: 195-210.

Hadley, R. D., and S. B. Kater (1983) Competence to form electrical connections is restricted to growing neurites in the snail, Helisoma. J. Neurosci. 3: 924-932.

Hadley, R. D., S. B. Kater, and C. S. Cohan (1983) Electrical synapse formation depends on interaction of mutually growing neurites. Science 221: 466-468.

Hadley, R. D., D. A. Bodnar, and S. B. Kater (1985) Formation of electrical synapses between isolated, cell cultured Helisoma neurons requires mutual neurite elongation. J. Neurosci. 5: 3145-3153.

Haydon, P. G., D. P. McCobb, and S. B. Kater (1984) Serotonin selectively inhibits growth cone motility and synaptogenesis of specific identified neurons. Science 226: 561-564.

Haydon, P. G., C. S. Cohan, D. P. McCobb, H. R. Miller, and S. B. Kater (1985) Neuron-specific growth cone properties as seen in identified neurons of Helisoma. J. Neurosci. Res. 13: 285-300.
Haydon, P. G., D. P. McCobb, and S. B. Kater (1987) The regulation of neurite outgrowth, growth cone motility, and electrical synaptogenesis by serotonin. J. Neurobiol. 18: 197-215.

Henderson, L. P. (1983) The role of 5-hydroxytryptamine as a transmitter between identified leech neurones in culture. J. Physiol. (Lond.) 339: 309-324.

Henderson, L. P., D. P. Kuffler, J. G. Nicholls, and R.-J. Zhang (1983) Structural and functional analysis of synaptic transmission between identified leech neurones in culture. J. Physiol. (Lond.) 340: 347-358.

Hume, R. I., L. W. Role, and G. D. Fischbach (1983) Acetylcholine release from growth cones detected with patches of acetylcholine receptor-rich membranes. Nature 305: 632-634.

Johnson, D. A., and G. Pilar (1980) The release of acetylcholine from postganglionic cell bodies in response to depolarization. J. Physiol. (Lond.) 299: 605-619.

Kostyuk, P. G. (1980) Calcium ionic channels in electrically excitable membrane. Neuroscience 5: 945-959.

Lemos, J. R., J. J. Nordmann, I. M. Cooke, and E. L. Stuenkel (1986) Single channels and ionic currents in peptidergic nerve terminals. Nature 319: 410-413.

Llinás, R., I. Z. Steinberg, and K. Walton (1981a) Presynaptic calcium currents in squid giant synapse. Biophys. J. 33: 289-322.

Llinás, R., I. Z. Steinberg, and K. Walton (1981b) Relationship between presynaptic calcium current and postsynaptic potential in squid giant synapse. Biophys. J. 33: 323-352.

Martin, A. R., and G. L. Ringham (1975) Synaptic transfer at a vertebrate central nervous system synapse. J. Physiol. (Lond.) 25l: 409426.

Pellegrino, M., and M. Simonneau (1984) Distribution of receptors for acetylcholine and 5-hydroxytryptamine on identified leech neurones growing in culture. J. Physiol. (Lond.) 352: 669-684.

Schacher, S., S. G. Rayport, and R. T. Ambron (1985) Giant Aplysia neuron R2 reliably forms strong chemical connections in vitro. $\mathrm{J}$. Neurosci. 5: 2851-2856.

Schuetze, S. M., and L. W. Role (1987) Developmental regulation of nicotinic acetylcholine receptors. Annu. Rev. Neurosci. 10:403-457.

Wojtowicz, J. M., and H. L. Atwood (1984) Presynaptic membrane potential and transmitter release at the crayfish neuromuscular junction. J. Neurophysiol. 52: 99-113.

Wong, R. G., R. D. Hadley, S. B. Kater, and G. C. Hauser (1981) Neurite outgrowth in molluscan organ and cell cultures: The role of conditioning factor(s). J. Neurosci. 1: 1008-1021.

Xie, Z. P., and M.-M. Poo (1986) Initial events in the formation of neuromuscular synapse: Rapid induction of acetylcholine release from embryonic neuron. Proc. Natl. Acad. Sci. USA 83: 7069-7073.

Young, S. H., and M.-M. Poo (1983) Spontaneous release of neurotransmitter from growth cones of embryonic neurones. Nature 305 : 634-637. 